\title{
A Numerical Investigation of Controllably Flexible Hydrofoil in Laminar Flows
}

\author{
G. Y. $\mathrm{He}^{1}$, X. Zhang ${ }^{2}$, S. G. Zhang ${ }^{1}$, G. W. He ${ }^{2}$ \\ ${ }^{1}$ The School of Aeronautic Science and Engineering, Beihang University, Beijing 100083, China \\ ${ }^{2}$ LNM, Institute of Mechanics, Chinese Academy of Sciences, Beijing 100080, China \\ Email: hgw@lnm.imech.ac.cn
}

\begin{abstract}
Aquatic animals, such as fishes, whales, seals and penguins, are naturally born to be flexible and deformable, which promise their effective locomotion through water. They are able to produce hydrodynamic thrust by active control of their body configurations. That is, the aquatic animals could wiggle their flexible bodies at an appropriate frequency and amplitude suitable to the hydrodynamics surrounding them. However, the mechanism for the active controls has not been adequately understood yet and attracts current research. One obstacle which hinders such investigation is the difficulty in experimental measurements of the flows around the wiggling bodies, and thus numerical simulation is becoming an indispensable alternative. In the paper, an immersed boundary method is developed to simulate the NACA 65-10 hydrofoil. It is observed that a wiggling hydrofoil exhibits a higher thrust while a stationary hydrofoil offers little improvement.
\end{abstract}

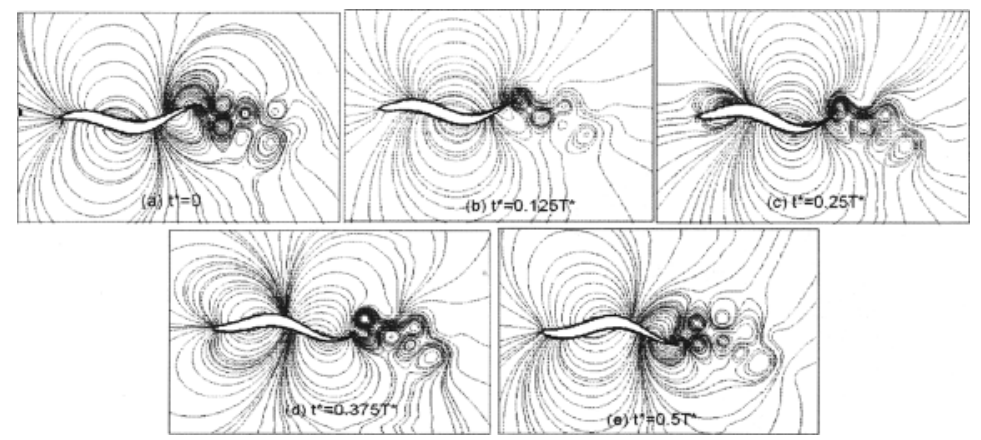

Figure: Time variation of the pressure contours $(R e=100)$

\section{REFERENCES}

1. Liu H. Simulation-based biological fluid dynamics in animal locomotion, Applied Mechanics Reviews, 2005; 58: 269-282

2. Lighthill M J. Note on the swimming of slender fish. J Fluid Mech, 1960;9: 305-317

3. Trianafyllou G S, Trianafyllou M S, Grosenbaugh M A. Optimal thrust development in oscillating foils with application to fish propulsive. J Fluids Struct, 1993; 7: 205-224

4. Trianafyllou M S, Trianafyllou G S. An efficient swimming machine. Sci Am, 1995; 3: 1-26

5. Akimoto H, Miyata H. Finite-volume simulation of a flow about a moving body with deformation. In: Proc. 5th Int. Symp. Comp. Fluid Dynamics, 1993; 1: 13-18

6. Mittal R, Iaccarino G. Immersed boundary methods. Annual Review of Fluid Mechanics, 2005; 37: 239-261

7. Kim J, Kim D, Choi H. An immersed-boundary finite-volume method for simulations of flow in complex geometries. J Comp Phys, 2001; 171: 132

8. Su S W, Lai M C, Lin C A. An immersed boundary technique for simulating complex flows with rigid boundary. Computer \& Fluids, September 2005 